\title{
NETS AND RECURRENCE IN TRANSFORMATION GROUPS
}

\author{
BY \\ F. J. HAHN( $\left(^{1}\right)$
}

1. Introduction. Problems concerning recurrence in a transformation group $(X, T)$, where $T$ is an abelian group, are discussed in $[1 ; 2 ; 4 ; 5]$. In the classical case where $T$ is the additive group of the real numbers we say $T$ is recurrent $(+)\{(-)\}$ at $x$ if there is a sequence $t_{n} \rightarrow+\infty\left\{t_{n} \rightarrow-\infty\right\}$ such that $\lim t_{n} x=x$. For more general groups Barbachine [1] has discussed recurrence under the assumption that the group is partially ordered. Gottschalk and Hedlund treat recurrence in terms of a distinguished class of semigroups of the group.

The classical notion, as previously described, treats recurrence in terms of sequences and therefore one has the feeling that a treatment for more general groups can be given in terms of nets and subnets. Such a treatment would appeal to those who feel at ease with convergence concepts.

$\S \S 2$ and 3 contain a brief discussion of orderings on groups and factor groups and display the machinery which will be used later. In $\$ 4$ a definition of recurrence is given in terms of nets and subnets. This definition brings us closer to the work of Barbachine but it is shown that it is equivalent to the definition given by Gottschalk and Hedlund. The concept of omega limiting sets is also defined. This section is ended with a discussion of the inheritance theorem for recurrence. Because of known material on nets a rather transparent proof of this theorem can be given. In $\$ 5$ special orbits are defined and the following theorem is proved: If $X$ is a compact space acted upon by a generative group $T$ and if $X$ is the closure of a recurrent trajectory then the set of special orbits of $X$ is either dense or empty. Throughout the paper $T$ is an abelian group.

2. Orderings and semigroups. By an ordering of $T$ we shall mean a set $R \subset T \times T$ such that $R$ is a semigroup under the composition $\left(s, s^{\prime}\right)\left(t, t^{\prime}\right)$ $=\left(s t, s^{\prime} t^{\prime}\right)$, and if $s \in T$ and $\left(t, t^{\prime}\right) \in R$ then $\left(s t, s t^{\prime}\right) \in R$. Instead of $\left(t, t^{\prime}\right) \in R$ we will of ten write $t<t^{\prime}(R)$ or $t<t^{\prime}$ if $R$ is clearly understood. It follows quickly that any such ordering is transitive.

Let $R$ be the collection of all orderings of $T$ and $\beta$ be the collection of all semigroups of $T$ and define the following maps:

This paper has been submitted to and accepted for publication by the Proceedings of the American Mathematical Society. It has been transferred to these Transactions, with the consent of the author, for technical reasons. Received by the editors July 25, 1960.

(1) National Science Foundation Fellow. 


$$
\begin{aligned}
& f: \odot \rightarrow R ; f(P)=\left\{(s, t): t s^{-1} \in P\right\}, \\
& g: R \rightarrow \odot ; g(R)=\{t:(e, t) \in R\},
\end{aligned}
$$

where $e$ is the group identity. It is easily verified that these mappings are well defined and that they are mutually inverse so each is one-to-one and onto.

We shall say that $T$ is directed by an ordering $R$ if each two elements of $T$ have an upper bound; that is if $s$ and $s^{\prime} \in T$ then there is a $t \in T$ such that $s<t$ and $s^{\prime}<t$.

THEOREM 1. If $R \in R$ and $g(R)=P$ then the following are equivalent:

(a) $T$ is directed by $R$.

(b) If $F \subset T$ is finite then there is a $t \in T$ such that $t F \subset P$.

(c) $P P^{-1}=T$ (see also $[5$, p. $57 ; 3$, p. 5]).

Proof. (a) implies (b). If $T$ is directed by $R$ and if $F$ is a finite set then $F^{-1}$ has an upper bound $t$ and thus $t F \subset P$.

(b) implies (c). If $s \in T$ then $s^{-1}$ and $s^{-2}$ have an upper bound $t$. Thus $t s$ and $t s^{2} \in P$ so $s=(t s)^{-1} t s^{2} \in P^{-1} P$.

(c) implies (a). If $s_{1}$ and $s_{2} \in T$ then $s_{i}=p_{i}^{-1} q_{i}$ where $p_{i}$ and $q_{i} \in P$ and $i=1$, 2. If we let $t=q_{1} q_{2}$ then $t s_{1}^{-1}=p_{1} q_{2} \in P$ and $t s_{2}^{-1}$ and $p_{2} q_{1} \in P$ so $t$ is an upper bound for $s_{1}$ and $s_{2}$.

In the preceding theorem the group $T$ might just as well always be discrete since its topology never enters into the argument. We now introduce the following notion: $T$ is strongly directed by $R \in R$ if each compact set has an upper bound. We also recall the following definition [4, p. 26]: a semigroup $P$ is replete if each compact subset of $T$ has a translate which is contained in $P$.

THEOREM 2. If $R \in R$ and $g(R)=P$ then the following are equivalent.

(a) $T$ is strongly directed by $R$,

(b) $P$ is a replete semigroup.

Proof. The proof uses the same techniques as Theorem 1 and is immediate.

A subset of $T$ is said to be extensive if it meets each replete semigroup [5, p. 26]. We will say a subset $S$ of $T$ is extensive with respect to an ordering $R$ if for each $t \in T$ there is an $s \in S$ such that $t<s(R)$ (e.g. $(t, s) \in R)$. If $P$ is a replete semigroup then Baum [2, p. 1146] defines a set to be $P$-extensive if for each $p \in P$ it meets $p P$. For the remainder of the paper we will restrict ourselves to orderings which strongly direct $T$.

Theorem 3. A subset $S$ of $T$ is extensive if and only if it is extensive with respect to each ordering of $T$.

Proof. If $S$ is extensive and $t \in T$ and $R \in R$ then $S$ meets every translate of $P=g(R)$ [5, p. 59] and in particular there is an $s \in t P \cap S$ so $t<s(R)$.

If $S$ is extensive with respect to each $R \in R$ and if $P$ is a replete semigroup 
we let $R=f(P)$. It follows that there is an $s \in S$ such that $e<s(R)$ and thus $s \in P$.

Corollary 4. A subset of $T$ is $P$-extensive if and only if it is extensive relative to the ordering $R=f(P)$.

A subset $S \subset T$ is said to be syndetic if there is a compact set $K \subset T$ such that $T=S K[5$, p. 12].

Theorem 5. A syndetic subset is extensive relative to all orderings of $T$.

Proof. This follows quickly from the fact that a syndetic subset is extensive $[5$, p. 59] and Theorem 3. We also give the proof in the language of orderings. Let $R \in R$ and $t_{0} \in T$. Since $T=S K$ we have $t_{0}=s_{0} k_{0}$, and $s_{0} K^{2}$ is compact so it has an upper bound $t=s k>s_{0} K^{2}(R)$. Thus $s>s_{0} k^{-1} K^{2}(R)$ and $s>s_{0} k^{-1} k k_{0}=s_{0} k_{0}=t_{0}$.

3. Orderings on factor groups. Let $S$ be a closed subgroup of $T$ and let $T^{\prime}=T / S$. $\odot^{\prime}$ will be the class of all semigroups of $T^{\prime}$ and $\mathbb{R}^{\prime}$ will be the class of all orderings of $T^{\prime}$. If $\phi$ is the natural map of $T$ onto $T^{\prime}$ then $\phi$ defines in an obvious manner mappings of $P$ onto $\mathcal{P}^{\prime}$ and $R$ onto $\mathcal{R}^{\prime}$. (In the second case $\phi$ is applied to each factor.) The mappings $f^{\prime}$ and $g^{\prime}$ are defined on $\boldsymbol{P}^{\prime}$ and $R^{\prime}$ in the same manner as before.

LEMMA 6. $f^{\prime} \phi=\phi f$ and $g^{\prime} \phi=\phi g$.

Proof. It is sufficient to prove $\phi=g^{\prime} \phi f$. If $R^{\prime}=\phi(R)$ and $R=f(P)$ then

$$
\begin{aligned}
g^{\prime} \phi f(P) & =g^{\prime} \phi(R) \\
& =g^{\prime}\left(R^{\prime}\right) \\
& =\left\{\phi(u):(\phi(e), \phi(u)) \in R^{\prime}\right\} \\
& =\{\phi(u): u \in P\}=\phi(P) .
\end{aligned}
$$

Lemma 7. If $R^{\prime}=\phi(R)$ then $\phi: T \rightarrow T^{\prime}$ is monotone with respect to the ordcrings $R$ and $R^{\prime}$. If $R=\phi^{-1}\left(R^{\prime}\right)$ then $\phi^{-1}$ is monotone with respect to these orderings in the sense that if $u<v\left(R^{\prime}\right)$ and if $s \in \phi^{-1}(u)$ and $t \in \phi^{-1}(v)$ then $s<t(R)$.

Proof. If $(s, t) \in R$ then $(\phi(s), \phi(t)) \in \phi(R)=R^{\prime}$. If $(u, v) \in R^{\prime}$ and $\phi(s)=u$, $\phi(t)=v$ then $(s, t) \in \phi^{-1}\left(R^{\prime}\right)=R$.

Corollary 8. $A \subset T$ is extensive $\{P$-extensive $\}$ if and only if $\phi(A) \subset T^{\prime}$ is extensive $\{\phi(P)$-extensive $\}$.

Proof. This follows from Lemma 7 and Theorem 3.

THEOREM 9. If $S$ is a finite $\{$ compact $\}$ subgroup of $T$ then the orderings which direct $\{$ strongly direct $\} T$ are taken by $\phi$ onto those orderings which direct \{strongly direct $\} T^{\prime}$ (see also [4, p. 23 problem 9]).

Proof. If $R$ directs $T$ and if $S \subset T$ and $F^{\prime} \subset T^{\prime}$ are finite then $F=\phi^{-1}\left(F^{\prime}\right)$ 
is finite and there is an upper bound $t$ of $F$ in $T$ with respect to $R$. It follows from Lemma 7 that $\phi(t)$ is an upper bound for $F^{\prime}$ with respect to $\phi(R)$, so $\phi(R)$ directs $T^{\prime}$.

If $R^{\prime}$ directs $T^{\prime}$ and $S$ is finite we let $R=\phi^{-1}\left(R^{\prime}\right)$ and we must show that $R$ directs $T$. If $F \subset T$ is finite then $\phi(F)=F^{\prime}$ is finite and has an upper bound $t^{\prime}$ in $T^{\prime}$ relative to $R^{\prime}$. If $t \in \phi^{-1}\left(t^{\prime}\right)$ then by Lemma $7 t$ is an upper bound for $\phi^{-1}\left(F^{\prime}\right) \supset F$ relative to $R$.

If $S$ is compact we need merely replace the word finite in the proof by the word compact.

4. Recurrent nets and limiting sets. Let $R$ be an ordering which directs $T$ and let $Y$ be a topological space. A map $F: T \rightarrow Y$ is a net which we will sometimes write as $F$ or $\{F(t): t \in T, R\}$.

Definition 10. A net $F: T \rightarrow Y$ is recurrent if for each ordering $R$ the net $F$ has a subnet converging to $F(e)$.

THEOREM 11. If $F: T \rightarrow Y$ then the following statements are equivalent:

(a) If $U$ is any neighborhood of $F(e)$ then there is an extensive set $A$ such that $F(A) \subset U$.

(b) For each neighborhood $U$ of $F(e)$ and each $t_{0} \in T$ and each ordering $R$ there is a $t>t_{0}(R)$ such that $F(t) \in U$.

(c) The net $F$ is recurrent.

Proof. (a) implies (b). Let $U$ be a neighborhood of $F(e)$ and let $t_{0} \in T$ and let $R$ be an ordering. By (a) there is an extensive set $A$ such that $F(A) \subset U$. Since $A$ is extensive there is a $t \in A$ such that $t>t_{0}(R)$ (Theorem 3).

(b) implies (c). Let $R$ be an ordering of $T$ and let $\mathcal{U}$ be the family of all neighborhoods of $F(e)$ directed as follows: $U<V$ if and only if $U \supset V$. Form the product directed set $T \times \mathfrak{u}[7$, p. 68] and define $G: T \times \mathfrak{u} \rightarrow T$ as follows: $G(t, U)=s$ where $s$ is any element of $T$ such that $s>t(R)$ and $F(s) \in U$. The subnet $F \circ G$ converges to $F(e)$.

(c) implies (a). Let $U$ be a neighborhood of $F(e)$ and let $R$ be any ordering of $T$ and for each $t_{0} \in T$ let $A\left(t_{0}, R\right)=\left\{t: t>t_{0}\right.$ and $\left.F(t) \in U\right\}$. Statement (c) insures us that $A\left(t_{0}, R\right)$ is not empty. If we let $A=\bigcup_{t \in T} \bigcup_{R \in \mathscr{R}} A(t, R)$ then it follows from Theorem 3 that $A$ is extensive and clearly $F(A) \subset U$.

If $(X, T)$ is a transformation group and if $Y=X$ and $x \in X$ the mapping $F: T \rightarrow X$ may be defined as $F(t)=t x$. In this case (a) of Theorem 11 is the definition of recurrence at $x[5, \mathrm{p} .26]$.

If we let $Y=\{0,1\}$ and have the discrete topology, and let $V$ be a neighborhood of $x$ we may define $F_{V}: T \rightarrow Y$ as follows: $F_{V}(t)=1$ if $t V \cap V \neq \varnothing$ and $F_{V}(t)=0$ if $t V \cap V=\varnothing$. In this case if (a) of Theorem 11 holds for each such $V$ then it is the definition of regional recurrence.

For the remainder of the paper we will consider a transformation group $(X, T)$ and the nets $F: T \rightarrow X$ will always be defined as $F(t)=t x$. 
THEOREM 12. If $S$ is a compact subgroup of $T$ then $T$ is recurrent at $x$ if and only if $T / S$ is recurrent at $S x$.

Proof. A proof of this may be found in [6] but we give here another proof which is quite natural in terms of convergence concepts. Let $(\psi, \phi)$ be the equivariant mapping of $(X, T)$ onto $(X / S, T / S)$. If $R$ is an ordering of $T$ and if $T$ is recurrent at $x$ there is a subnet $\{t(n) x: n \in N, t(n) \in T, R\}$ of $\{t x: t \in T, R\}$ such that $t(n) x \rightarrow x$. Thus $\phi(t(n)) \psi(x)=\psi(t(n) x) \rightarrow \psi(x)$ and it follows from Lemma 7 that $\{\phi(t(n)) \psi(x)\}$ is a subnet of

$$
\{\phi(t) \psi(x): \phi(t) \in T / S, \phi(R)\} .
$$

If there is a subnet $\{\phi(t(n)) \psi(x)\}$ of $\{\phi(t) \psi(x): \phi(t) \in T / S, \phi(R)\}$ such that $\phi(t(n)) \psi(x) \rightarrow \psi(x)$ then there exists a net $t(n) k(n) x \rightarrow x$ where $t(n) \in T$ and $k(n) \in S$ and $\{t(n) k(n)\}$ is extensive. Since $S$ is compact there is a subnet $\{k(m)\}$ of $\{k(n)\}$ such that $k(m) \rightarrow k$. It follows that $k t(m) x \rightarrow x$. Using Lemma 7 and the fact that $t(m) k(m)$ and $t(m) k$ are in the same coset it is not hard to see that $\{k t(m) x\}$ is a subnet of $\{t x: t \in T ; R\}$.

DEFInition 13. The omega limiting set of $x \in X$ with respect to the ordering $R$ is the set of all points $y \in X$ such that the net $\{t x: t \in T, R\}$ has a subnet which converges to $y$ and is denoted by $\Omega(x, T, R)$.

We need the following theorem and its corollary for later reference.

TheOREM 14 (TheOREM ON ITERATED Limits). Let $M$ be a directed set, let $N_{m}$ be a directed set for each $m$ in $M$, let $L$ be the product directed set $M \times \prod\left\{N_{m}: m \in M\right\}$ and for $(m, f) \in L$ let $H(m, f)=(m, f(m))$. If $G(m, n)$ is a member of a topological space for each $m \in M$ and $n \in N_{m}$ and if $\lim _{m} \lim _{n} G(m, n)$ exists then the net $\{G H(m, f):(m, f) \in L\}$ converges to this limit $[7$, p. 69].

Corollary 15. Let $R$ be an ordering of $T$, let $M$ and $N$ be directed sets, let $r: M \rightarrow T$ and $s: N \rightarrow T$ be subnets of the net $\{t x: t \in T, R\}$, let $L$ be the product directed set $M \times N^{M}$ and for each $(m, f) \in L$ let $j(m, f)=r(m) s(f(m))$. If $\lim _{m} r(m) x=r_{0} x$ and $\lim _{n} s(n) x=s_{0} x$ then $j: L \rightarrow T$ is a subnet of the net $\{t x: t \in T, R\}$ and $\lim _{l} j(l) x=s_{0} r_{0} x$.

Proof. Since $\lim _{m} \lim _{n} r(m) s(n) x=\lim _{m} r(m) s_{0} x=r_{0} s_{0} x$ we need only apply the preceding theorem and make the straightforward observation that $j$ is a subnet.

DEFINITION 16. If $S \subset T$ is a syndetic subgroup and if $R$ is an ordering of $T$ then the omega- $x$ envelope of $S$ with respect to $R$, denoted by $S(R, x)$, is the subset $\{t: t x \in \Omega(x, S, R)\}$.

Lemma 17. If $S \subset T$ is a syndetic subgroup and $R$ is an ordering of $T$ and $S(R, x) \neq \varnothing$ then $S(R, x)$ is a closed syndetic subgroup of $T$.

Proof. From Theorem 14 it follows that $S(R, x)$ is closed. If $t \in S(R, x)$ then clearly $t S \subset S(R, x)$ so $S(R, x)$ is syndetic. Corollary 15 shows that 
$S(R, x)$ is a semigroup and since any syndetic semigroup is a subgroup [5, p. 12] the theorem is complete.

In $\left[5\right.$, p. 13] the $x$-envelope of $S$, denoted by $S_{x}$, is defined as the subset $\{t: t x \in[S x]-\}$. Clearly it is always the case that $S(R, x) \subset S_{x}$. If $S$ is recurrent at $x$ relative to $R$ then the opposite inclusion follows from the theorem on iterated limits and $S(R, x)=S_{x}$. If the two sets are equal then $S(R, x) \neq \varnothing$ so it is a group and contains $e$. Thus $S$ is recurrent at $x$ relative to $R . S(R, x)$ has the choice of either being empty or equal to $S_{x}$. These observations give a transparent proof for the following known theorem [5, p. 64].

THEOREM 18. If $S \subset T$ is a syndetic subgroup then $T$ is recurrent at $x$ if and only if $S$ is recurrent at $x$.

Proof. $S$ recurrent at $x$ implies $T$ recurrent at $x$ since $S$ is syndetic. Let $R$ be any ordering of $T$, let $T=K S$ where $K$ is compact and let $T$ be recurrent at $x$ with respect to $R$. There is a subnet $t: N \rightarrow T$ of $\{t x: t \in T, R\}$ such that $\lim _{n} t(n) x=x$. If we let $t(n)=k(n) s(n)$ where $k(n) \in K$ and $s(n) \in S$ then since $K$ is compact there is a subnet $r: M \rightarrow N$ such that $\lim _{m} k(r(m))=k$. Thus $x=\lim _{m} s(r(m)) k(r(m)) x=k \lim _{m} s(r(m)) x$ so $k^{-1} \in S(R, x)$ which is nonempty implying that $S$ is recurrent at $x$ with respect to $R$.

If in the proof of the inheritance theorem for regional recurrence $[5$, p. 67] we use the following easily proved lemma: If $K \subset T$ is compact and $S \subset T$ and $s_{0} \in S$ then there is a $t_{0}$ such that $t>t_{0}$ and $s \in t K \cap S$ implies $s>s_{0}$; in place of Theorem $6.20[5, \mathrm{p} .60]$ then the proof is analogous to the one which would be given if $T$ were the additive group of the reals.

5. Nonrecurrent trajectories in a compact space. Throughout this section we consider a transformation group $(X, T)$ where $X$ is a compact Hausdorff space (thus a uniform space) and $T$ is a generative group; that is $T$ is locally compact, abelian, and generated by a compact neighborhood of the identity. It is known $[8, \mathrm{p} .110]$ that $T=K \times{g^{p}}^{p} \times \mathcal{S}^{q}$ where $K$ is a compact abelian group, $g$ is the integers, $S$ is the reals, and $p$ and $q$ are non-negative integers.

Definition 19. An ordering $R$ of $T$ will be said to be a real ordering if the following are satisfied.

(a) If $t_{0}, t_{1} \in T$ then $\left\{t: t<t_{1}\right\} \cap\left\{t: t>t_{0}\right\}$ is compact.

(b) If $t_{0} \in T$ then $\left\{t: t>t_{0}\right\}$ is closed.

(c) If $F \subset T$ is a closed subset which is bounded from below then there is a $t \in F$ such that $s<t$ and $s \neq t$ implies $s \notin F$. (Such an element $t$ in $F$ will be called an infimum of $F$.)

LEMMA 20. If $T=S^{a}$ and $P^{\prime} \subset T$ is a replete semigroup then there is a replete semigroup $P \subset P^{\prime}$ such that $R=f(P)$ is a real ordering.

Proof. It is known [6] that $P^{\prime}$ contains a replete semigroup $p+P$ where $p \in P$ and $P=\left\{\sum_{i=1}^{a} a_{i} t_{i}: a_{i} \geqq 0,\left\{t_{i}\right\}\right.$ is linearly independent $\}$. It is not too difficult to see that if $f(P)$ is a real ordering then so is $f(p+P)$. We will show that $f(P)$ is a real ordering. 
(a) Since the set $\left\{t: t<t_{2}\right\} \cap\left\{t: t>t_{1}\right\}=t_{2}-P \cap t_{1}+P$ we need only show that $t+P \cap-P$ is compact for $t \in T$. If $t=\sum b_{i} t_{i}$ then $t+P \cap-P$ $=\left\{\sum a_{i} t_{i}: b_{i} \leqq a_{i} \leqq 0\right\}$ which is compact.

(b) The set $P$ is closed; thus so is $t_{0}+P$ and so is $\left\{t: t>t_{0}\right\}$.

(c) Because of the translation properties of the ordering $f(P)$ we may assume $e<F$. Let $T$ be normed so that if $t=\sum b_{i} t_{i}$ then $\|t\|=\sum\left|b_{i}\right|$. Choose $t \in F, t=\sum b_{i} t_{i}$, such that $\|t\|$ is minimal. To prove (c) we need only examine those $s$ such that $s \neq t, s<t$ and $s \in P$ (since $F \subset P$ ). That is if $s=\sum a_{i} t_{i}$ then $b_{i} \geqq a_{i} \geqq 0, i=1,2, \cdots, q$ and $b_{j}>a_{j}$ for at least one $j$. But for each such $s$ we have $\|s\|<\|t\|$ so $s \notin F$.

Lemma 21. If $T=g^{p} \times S^{q}$ and $P^{\prime} \subset T$ is a replete semigroup then there is a replete semigroup $P \subset P^{\prime}$ such that $f(P)$ is a real ordering.

Proof. Consider $T$ imbedded in $T^{\prime}=\mathcal{S}^{p+q}$. There is a replete semigroup $Q$ in $T^{\prime}$ such that $T \cap Q \subset P^{\prime}[5$, p. 58]. $Q$ contains a closed replete semigroup $Q^{\prime}$ in $T^{\prime}$ which yields a real ordering for $T^{\prime}$. If we let $T \cap Q^{\prime}=P \subset P^{\prime}$ the theorem is complete.

Lemma 22. Let $T$ be generative, and let $T=K \times \mathcal{g}^{p} \times \mathcal{S}^{q}$ and let $\phi: T \rightarrow T / K$ be the natural map. If $P^{\prime} \subset T$ is a replete semigroup then $P^{\prime}$ contains a replete semigroup $P$ such that the ordering $f(\phi(P))$ on $T / K$ is a real ordering.

Proof. $P^{\prime}$ contains a replete semigroup $Q$ such that $K Q \subset P^{\prime}$ [6]. It follows from the previous lemma that there is a replete semigroup $Q^{\prime} \subset \phi(Q)$ which induces a real ordering on $T / K . P=\phi^{-1}\left(Q^{\prime}\right) \subset K Q \subset P^{\prime}$ serves the purpose of the theorem.

LemMA 23. If $T$ is any group and $K$ is a compact subgroup and $\phi: T \rightarrow T / K$ is the natural map then $A$ is dense in $T$ if and only if $\phi(A)$ is dense in $T / K$.

Proof. This follows from the fact that $\phi$ is closed and continuous.

Definition 25. A point $x \in X$ will be said to lie on a special trajectory if there is an ordering $R$ of $T$ such that $\Omega(x, T, R) \neq X$.

TheOREM 24. Let $X$ be an orbit closure of a recurrent trajectory. If $X$ has one point lying on a special trajectory then the points which lie on special trajectories are dense in $X$.

Proof. If $T$ is compact $X$ has no points on special trajectories so we consider $T$ to be noncompact. By using the techniques of Theorem 12 we can easily show that if $R$ is an ordering of $T$ and if $(\psi, \phi)$ is the natural map of $(X, T)$ onto $(X / K, T / K)$ then $\Omega(\psi(x), \phi(T), \phi(R))=\psi(\Omega(x, T, R))$. Thus, Theorem 12 and Lemma 23 tell us we need only consider the transformation group $(X / K, T / K)$. Lemma 22 tells us that on $T / K$ we need only consider real orderings. We may thus reduce the problem to the case where $X$ is the closure of a recurrent trajectory and on $T$ we consider only real orderings. 
We suppose the theorem false. Let $R$ be a real ordering of $T$ and $z$ be a point such that $\Omega(z, T, R) \neq X$. There is a point $x$ which has a recurrent dense trajectory, and a neighborhood $V(x)$, and a $t_{0} \in T$ such that if $t>t_{0}(R)$ then $t z \notin V(x)$. For brevity we let $y=t_{0} z$ and then if $t>e(R)$ we have $t y \notin V(x)$. Since $T$ is recurrent at $x$ the orbit closure is equal to $\Omega(x, T, R)$ so there is a subnet $t: N \rightarrow T$ such that $\lim _{n} t(n) x=y$.

Let $W$ be an open entourage such that $W^{2} \subset V$ and let $A(n)$ $=\left\{t: t x \in[W(x)]^{-}\right.$and $\left.t>t(n)\right\}$. Each $A(n)$ is closed, nonempty and bounded from below so it has an infimum which we denote by $t^{\prime}(n)$. Since $X$ is compact we may assume that $\lim _{n} t^{\prime}(n) x=x_{0} \in[W(x)]-C V(x)$. Let $s: N \rightarrow T$ be defined by $s(n)=t^{\prime}(n) t^{-1}(n)$. We will show that $s$ is a subnet of the net $\{t: t \in T, R\}$. If $s$ is not a subnet then there is a $t_{1} \in T$ and a mapping $r: N \rightarrow N$ such that $r(n)>n(R)$ and $s(r(n))<t_{1}$. Since the net $s(r(n))$ is bounded from below by $e$ and from above by $t_{1}$ it has a subnet which converges. For ease of notation we assume that the net itself converges and $\lim _{n} s(r(n))=t_{2}>e(R)$. If we let $y(n)=t(r(n)) x$ then $t_{2} y=\lim _{n} s(r(n)) y(n)=\lim _{n} t^{\prime}(r(n)) x=x_{0}$. But this is impossible since $x_{0} \in V(x)$ and $t_{2}>e$ implies $t_{2} y \notin V(x)$.

If $t<e$ and $t \neq e$ then for $n$ sufficiently large we have $t^{\prime}(n)>t t^{\prime}(n)>t(n)$ and $t^{\prime}(n) \neq t t^{\prime}(n)$ so $t t^{\prime}(n) x \notin[W(x)]-$. Since $\lim _{n} t t^{\prime}(n) x=t x_{0}$ we see that $t x_{0} \notin W(x)$ for $t<e$ and $t \neq e$. Thus we have $x \in \Omega\left(x_{0}, T, R^{-1}\right)$. So $x_{0} \in V(x)$ has a special trajectory which is impossible since $V(x)$ was assumed to be free of special trajectories.

An immediate consequence is the fact that if a compact set which is the closure of a recurrent trajectory is not minimal then the points on special trajectories are dense.

\section{BIBLIOGRAPHY}

1. E. Barbachine, Sur le conducte des points sous les transformations homéomorphes de l'espace, C. R. (Doklady) Acad. Sci. USSR, vol. 51 (1946) pp. 3-5.

2. J. D. Baum, P-recurrence in topological dynamics, Proc. Amer. Math. Soc. vol. 7 (1956) pp. $1146-1154$. ter VI.

3. N. Bourbaki, Eléments de mathématique, XIV, Livre II, Paris, Hermann, 1952, Chap-

4. W. H. Gottschalk and G. A. Hedlund, The dynamics of transformation groups, Trans. Amer. Math. Soc. vol. 65 (1949) pp. 348-359.

5. - Topological dynamics, Amer. Math. Soc. Colloquium Publications, vol. 36, 1955.

6. F. Hahn, On some embeddings, recurrence properties, and the Birkhoff-Markov theorem for transformation groups, Duke Math. J. vol. 27 (1960) pp. 513-526.

7. J. L. Kelley, General topology, New York, D. Van Nostrand Co., 1955.

8. A. Weil, L'intégration dans les groupes topologiques et ses applications, Paris, Hermann, 1940.

INSTITUTE FOR ADVANCED STUdy, Princeton, New Jersey 